\title{
Stochastic Modeling of Nonlinear Random Vibrations Using Heavy-tailed Mixture Distribution*
}

\author{
Katsutoshi YoshidA $^{\dagger}$ and Yoshikazu YamanaKA ${ }^{\dagger}$
}

\begin{abstract}
In this study, we propose a simple probability density function (PDF) model of GaussianLaplacian mixture (GLM) type, which provides a concise parameterization of heavy-tailed data. We construct our model as convex combination of Gaussian and Laplacian PDFs to obtain a minimal parameterization of heavy-tailed data. We then conduct least-squares fitting of our model to a heavy-tailed data generated by a random Duffing oscillator and obtain over $94 \%$ of residual sum of squares (RSS) fitness. The resulting model is applied to predicting transient moment responses and achieves over $90 \%$ of RSS fitness to Monte-Carlo simulation results of the original system.
\end{abstract}

\section{Introduction}

Random vibrations in the real world sometimes exhibit heavy-tailed fluctuations whose PDFs have heavier tails than the Gaussian PDF. Huge amount of physical examples can be found in the fields of earth dynamics[1], economic dynamics[2], human dynamics[3-5], and so on.

From an engineering point of view, how to design and control such heavy-tailed fluctuations will become an important issue. However, calculating heavytailed PDFs is not necessarily easy task. One reason is that, in general, their explicit mathematical expressions are hardly obtainable as well as their covariances can be mathematically infinite[6]. Of course, numerical methods[7] are effective for such problems; however, if closed expressions of heavy-tailed PDFs are available even approximately, further engineering applications will follow.

In this study, we propose a simple PDF model of GLM type, which is capable of concisely parameterizing heavy-tailed data. The resulting PDF model is applied to prediction of transient moment responses of the original system.

The rest of the paper is constructed as follows. In Sec. 2, we describe an example of heavy-tailed PDF data observed in a random Duffing oscillator. In Sec. 3, we propose the GLM model of the PDF data. In Sec. 4, we fit our GLM model to the PDF data. In

* Manuscript Received Date: June 14, 2019

* The material of this paper was partially presented at the 50th ISCIE International Symposium on Stochastic Systems Theory and Its Applications(SSS '18) which was held in November, 2018.

$\dagger$ Department of Mechanical and Intelligent Engineering, Utsunomiya University; Yoto 7-1-2, Utsunomiya, Tochigi 321-8585, JAPAN
Sec. 5, we apply the fitted GLM model to prediction of transient moment responses of the original system. Sec. 6 concludes our study.

\section{Heavy-tailed Nonlinear Vibra- tion}

To provide a simple example, we consider a random Duffing oscillator in the following form:

$$
\ddot{x}+c \dot{x}+x+b x^{3}=s_{a} w_{1}(t)+s_{m} w_{2}(t) x,
$$

where $\dot{x}:=d x / d t, w_{1}(t)$ and $w_{2}(t)$ are independent Gaussian white noises with zero mean and unit variance, and $s_{a}$ and $s_{m}$ are additive and multiplicative intensities, respectively. By using a state vector $\boldsymbol{x}=\left(x_{1}, x_{2}\right)^{T}:=(x, \dot{x})^{T}\left({ }^{T}\right.$ denotes transpose $)$, eq. (1) can be rewritten in the first-order form:

$$
\begin{aligned}
& \dot{\boldsymbol{x}}=\boldsymbol{f}(\boldsymbol{x})+G(\boldsymbol{x}) \boldsymbol{w}(t), \\
& \boldsymbol{f}(\boldsymbol{x}):=\left(x_{2},-x_{1}-b x_{1}^{3}-c x_{2}\right)^{T}, \\
& G(\boldsymbol{x}):=\left[\begin{array}{cc}
0 & 0 \\
s_{a} & s_{m} x_{1}
\end{array}\right], \quad \boldsymbol{w}:=\left(w_{1}, w_{2}\right)^{T} .
\end{aligned}
$$

In Fig. 1, the small circles indicate a PDF data generated by eq. (2) for $b=1, c=0.7, s_{a}^{2}=0.01$, and $s_{m}^{2}=0.99$ (i.e., the multiplicative term is dominant), plotted as its marginal PDFs with respect to $x_{1}$ and $x_{2}$. The PDF data was obtained by normalizing twodimensional histogram of $10^{8}$ sample paths of eq. (2) at $t=50 \mathrm{~s}$, after their transient responses decayed. The sample paths are generated along separate sample paths of $w_{1}(t)$ and $w_{2}(t)$. The histogram was build on $51 \times 51$ uniform grid points inscribed in the rectangle domain $\left(x_{1}, x_{2}\right) \in[-0.5,0.5] \times[-0.5,0.5]$.

Note that, the stochastic processes generated by nonlinear stochastic systems generally depend on their initial states; however, the abovementioned parameter condition results in unimodal PDFs and 


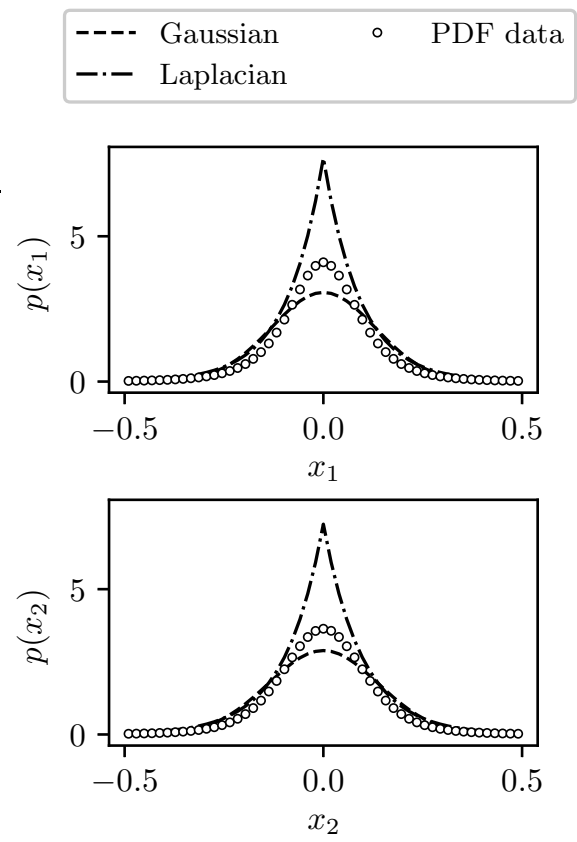

Fig. 1 Heavy-tailed PDF data versus Gaussian and Laplacian PDFs, plotted as their marginal PDFs with respect to $x_{1}$ and $x_{2}$. All PDFs have common mean and variance.

causes little dependency on initial states.

Furthermore, the broken and dashed lines in Fig. 1 indicate the corresponding one-dimensional Gaussian and Laplacian PDFs, respectively, whose mean and variance values are set to those obtained from the PDF data.

Fig. 1 clearly shows that the PDF data is not fitted well by both Gaussian and Laplacian PDFs and exhibits an intermediate feature between them. In particular, the PDF data yields significantly lower peaks near the origin than does the Laplacian PDF and higher peaks than does the Gaussian PDF. These are caused by coexistence of additive and multiplicative terms $\left(s_{a}, s_{m} \neq 0\right)$ and such distributions are easily found in real-world random behaviors [3,5].

\section{GLM Representation of Heavy- Tailed Density Functions}

As shown in Fig. 1, the vibration system in eq. (2) can produce an intermediate distribution between Gaussian and Laplacian. To describe this, we propose a GLM representation of PDFs.

\subsection{General Formulation}

Our proposed GLM-type PDF model is given in the following form:

$$
\begin{aligned}
& p_{\text {mix }}\left(\boldsymbol{x} ; \boldsymbol{\mu}, \boldsymbol{\Sigma}_{\mathcal{N}}, \boldsymbol{\Sigma}_{\mathcal{L}}, \kappa\right):= \\
& \quad \kappa \mathcal{N}\left(\boldsymbol{x} ; \boldsymbol{\mu}, \boldsymbol{\Sigma}_{\mathcal{N}}\right)+(1-\kappa) \mathcal{L}\left(\boldsymbol{x} ; \boldsymbol{\mu}, \boldsymbol{\Sigma}_{\mathcal{L}}\right) .
\end{aligned}
$$

Here, $\mathcal{N}$ and $\mathcal{L}$ are $n$-dimensional Gaussian[8] and Laplacian[9] PDFs, respectively, defined below. $\boldsymbol{\Sigma}_{\mathcal{N}}$ and $\boldsymbol{\Sigma}_{\mathcal{L}}$ denote respective covariance matrices, $\boldsymbol{\mu}$ a common mean vector, and $0 \leq \kappa \leq 1$ a mixture-ratio. The convex combination used in eq. (3) guarantees

$$
\int_{-\infty}^{\infty} p_{\operatorname{mix}}\left(\boldsymbol{x} ; \boldsymbol{\mu}, \boldsymbol{\Sigma}_{\mathcal{N}}, \boldsymbol{\Sigma}_{\mathcal{L}}, \kappa\right) d \boldsymbol{x}=1
$$

Here, the $n$-dimensional Gaussian distribution[8] is well known to be given by

$$
\begin{aligned}
& \mathcal{N}(\boldsymbol{x} ; \boldsymbol{\mu}, \boldsymbol{\Sigma}):=\frac{1}{(2 \pi)^{n / 2}}|\boldsymbol{\Sigma}|^{-1 / 2} \\
& \quad \times \exp \left\{-\frac{1}{2}(\boldsymbol{x}-\boldsymbol{\mu})^{T} \boldsymbol{\Sigma}^{-1}(\boldsymbol{x}-\boldsymbol{\mu})\right\} .
\end{aligned}
$$

On the other hand, one can find different definitions of $n$-dimensional Laplace distributions $[9,10]$. In this study, we employ a finite-valued type of them, in the following form $[9]$ :

$$
\begin{aligned}
& \mathcal{L}(\boldsymbol{x} ; \boldsymbol{\mu}, \boldsymbol{\Sigma}):=\frac{(n+1) \Gamma(n / 2)}{(2 \pi)^{n / 2} \Gamma(n)}|\boldsymbol{\Sigma}|^{-1 / 2} \\
& \quad \times \exp \left\{-\sqrt{(n+1)(\boldsymbol{x}-\boldsymbol{\mu})^{T} \boldsymbol{\Sigma}^{-1}(\boldsymbol{x}-\boldsymbol{\mu})}\right\},
\end{aligned}
$$

where $\Gamma$ denotes the gamma function. The Laplace distribution in eq. (6) provides a rare example of heavy-tailed distribution which is able to be parameterized by mean $\boldsymbol{\mu}$ and covariance $\boldsymbol{\Sigma}$ only.

The characteristic functions of eqs. (5), (6), respectively, are given in the following forms[9]:

$$
\begin{aligned}
& \psi_{\mathcal{N}}(\boldsymbol{t} ; \boldsymbol{\mu}, \boldsymbol{\Sigma})=\exp \left\{i \boldsymbol{t}^{T} \boldsymbol{\mu}\right\} \exp \left\{-\frac{1}{2} \boldsymbol{t}^{T} \boldsymbol{\Sigma} \boldsymbol{t}\right\}, \\
& \psi_{\mathcal{L}}(\boldsymbol{t} ; \boldsymbol{\mu}, \boldsymbol{\Sigma})=\exp \left\{i \boldsymbol{t}^{T} \boldsymbol{\mu}\right\}\left(1+\frac{\boldsymbol{t}^{T} \boldsymbol{\Sigma} \boldsymbol{t}}{n+1}\right)^{-\frac{n+1}{2}},
\end{aligned}
$$

where $\boldsymbol{t}$ is an $n$-dimensional vector and $i:=\sqrt{-1}$. Respective moment-generating functions are given by

$$
\begin{aligned}
& M_{\mathcal{N}}(\boldsymbol{t} ; \boldsymbol{\mu}, \boldsymbol{\Sigma}):=\psi_{\mathcal{N}}(-i \boldsymbol{t} ; \boldsymbol{\mu}, \boldsymbol{\Sigma}), \\
& M_{\mathcal{L}}(\boldsymbol{t} ; \boldsymbol{\mu}, \boldsymbol{\Sigma}):=\psi_{\mathcal{L}}(-i \boldsymbol{t} ; \boldsymbol{\mu}, \boldsymbol{\Sigma}) .
\end{aligned}
$$

Note that, unfortunately, the marginal PDF of the multi-dimensional Laplace PDF in eq. (6) does not match $\left.\mathcal{L}(\boldsymbol{x} ; \boldsymbol{\mu}, \boldsymbol{\Sigma})\right|_{n=1}$. This consistency problem seems still open in the field of heavy-tailed distributions. Therefore, in our numerical examples below, we obtain one-dimensional marginal plots of multidimensional PDFs by numerically integrating them.

\subsection{Two-dimensional Case}

Hereafter, $\langle\cdots\rangle$ denotes an ensemble average, $\mu_{i}:=$ $\left\langle x_{i}\right\rangle$ denotes a mean, and $\sigma_{i j}:=\left\langle\left(x_{i}-\mu_{i}\right)\left(x_{j}-\mu_{j}\right)\right\rangle$ denotes a covariance, for $1 \leq i \leq j \leq 2$.

In two-dimensional case $(n=2)$, eqs. (5), (6), (9), (10) can be written down in the following forms, respectively,

$$
\mathcal{N}\left(x_{1}, x_{2} ; \boldsymbol{\mu}, \boldsymbol{\Sigma}\right)=\frac{1}{2 \pi \sqrt{C}} \exp \left\{-\frac{Q}{2 C}\right\},
$$




$$
\mathcal{L}\left(x_{1}, x_{2} ; \boldsymbol{\mu}, \boldsymbol{\Sigma}\right)=\frac{3}{2 \pi \sqrt{C}} \exp \left\{-\sqrt{\frac{3 Q}{C}}\right\},
$$

where $\quad C:=\sigma_{11} \sigma_{22}-\sigma_{12}^{2}, \quad Q:=\sigma_{22}\left(x_{1}-\mu_{1}\right)^{2}-$ $2 \sigma_{12}\left(x_{1}-\mu_{1}\right)\left(x_{2}-\mu_{2}\right)+\sigma_{11}\left(x_{2}-\mu_{2}\right)^{2}$, and

$$
\begin{gathered}
M_{\mathcal{N}}\left(t_{1}, t_{2} ; \boldsymbol{\mu}, \boldsymbol{\Sigma}\right)=\exp \left\{\mu_{1} t_{1}+\mu_{2} t_{2}\right\} \\
\quad \times \exp \left\{\frac{\sigma_{11} t_{1}^{2}+2 \sigma_{12} t_{1} t_{2}+\sigma_{22} t_{2}^{2}}{2}\right\}, \\
M_{\mathcal{L}}\left(t_{1}, t_{2} ; \boldsymbol{\mu}, \boldsymbol{\Sigma}\right)=\exp \left\{\mu_{1} t_{1}+\mu_{2} t_{2}\right\} \\
\quad \times\left(1-\frac{\sigma_{11} t_{1}^{2}+2 \sigma_{12} t_{1} t_{2}+\sigma_{22} t_{2}^{2}}{3}\right)^{-3 / 2} .
\end{gathered}
$$

In this case, GLM model in eq. (3) becomes

$$
\begin{gathered}
p_{\text {mix }}\left(x_{1}, x_{2} ; \boldsymbol{\mu}, \boldsymbol{\Sigma}_{\mathcal{N}}, \boldsymbol{\Sigma}_{\mathcal{L}}, \kappa\right):=\kappa \mathcal{N}\left(x_{1}, x_{2} ; \boldsymbol{\mu}, \boldsymbol{\Sigma}_{\mathcal{N}}\right) \\
+(1-\kappa) \mathcal{L}\left(x_{1}, x_{2} ; \boldsymbol{\mu}, \boldsymbol{\Sigma}_{\mathcal{L}}\right), \quad 0 \leq \kappa \leq 1 .
\end{gathered}
$$

\section{GLM Fitting to PDF Data}

Let $\bar{p}\left(x_{1}, x_{2}\right)$ denote the PDF data shown in Fig. 1 whose mean and covariance are written as

$$
\overline{\boldsymbol{\mu}}=\left[\begin{array}{l}
\bar{\mu}_{1} \\
\bar{\mu}_{2}
\end{array}\right], \quad \overline{\boldsymbol{\Sigma}}=\left[\begin{array}{ll}
\bar{\sigma}_{11} & \bar{\sigma}_{12} \\
\bar{\sigma}_{12} & \bar{\sigma}_{22}
\end{array}\right] .
$$

In this section, we consider how to obtain the bestfitting $p_{\text {mix }}\left(x_{1}, x_{2} ; \boldsymbol{\mu}, \boldsymbol{\Sigma}_{\mathcal{N}}, \boldsymbol{\Sigma}_{\mathcal{L}}, \kappa\right)$ to $\bar{p}\left(x_{1}, x_{2}\right)$ in terms of least squares.

\subsection{Parameterization of $p_{\text {mix }}$}

We introduce a specification for fitting as follows.

- The fitted $p_{\text {mix }}$ has the same values of mean and covariance as those of $\bar{p}\left(x_{1}, x_{2}\right)$.

As for mean values, this specification can easily be fulfilled by substituting $\boldsymbol{\mu}=\overline{\boldsymbol{\mu}}$ into $p_{\text {mix }}$ of eq. (15).

On the other hand, as for covariance values, some other steps are required. Let $\boldsymbol{\Sigma}_{\text {mix }}$ denote a covariance matrix of $p_{\text {mix }}$; this is calculated as

$$
\begin{aligned}
\boldsymbol{\Sigma}_{\text {mix }} & =\iint_{-\infty}^{\infty} \boldsymbol{x} \boldsymbol{x}^{T} p_{\text {mix }}\left(x_{1}, x_{2} ; \boldsymbol{\mu}, \boldsymbol{\Sigma}_{\mathcal{N}}, \boldsymbol{\Sigma}_{\mathcal{L}}, \kappa\right) d x_{1} d x_{2} \\
= & \kappa \iint_{-\infty}^{\infty} \boldsymbol{x} \boldsymbol{x}^{T} \mathcal{N}\left(x_{1}, x_{2} ; \boldsymbol{\mu}, \boldsymbol{\Sigma}_{\mathcal{N}}\right) d x_{1} d x_{2} \\
& +(1-\kappa) \iint_{-\infty}^{\infty} \boldsymbol{x} \boldsymbol{x}^{T} \mathcal{L}\left(x_{1}, x_{2} ; \boldsymbol{\mu}, \boldsymbol{\Sigma}_{\mathcal{L}}\right) d x_{1} d x_{2} \\
= & \kappa \boldsymbol{\Sigma}_{\mathcal{N}}+(1-\kappa) \boldsymbol{\Sigma}_{\mathcal{L}}
\end{aligned}
$$

Thus, we can write the covariance matrix as

$$
\overline{\boldsymbol{\Sigma}}=\boldsymbol{\Sigma}_{\text {mix }}=\kappa \boldsymbol{\Sigma}_{\mathcal{N}}+(1-\kappa) \boldsymbol{\Sigma}_{\mathcal{L}} .
$$

Next, we introduce a covariance-ratio $\lambda_{i j}$ and put

$$
\left(\boldsymbol{\Sigma}_{\mathcal{N}}\right)_{i j}=\lambda_{i j}\left(\boldsymbol{\Sigma}_{\mathcal{L}}\right)_{i j}, \quad 1 \leq i \leq j \leq 2,
$$

where $(\cdot)_{i j}$ denotes $(i, j)$-element of a matrix. As $(\overline{\boldsymbol{\Sigma}})_{i j}=\kappa \lambda_{i j}\left(\boldsymbol{\Sigma}_{\mathcal{L}}\right)_{i j}+(1-\kappa)\left(\boldsymbol{\Sigma}_{\mathcal{L}}\right)_{i j}$, we obtain

$$
\left(\boldsymbol{\Sigma}_{\mathcal{N}}\right)_{i j}=\frac{\lambda_{i j}(\overline{\boldsymbol{\Sigma}})_{i j}}{\kappa \lambda_{i j}+(1-\kappa)},
$$

Table 1 GLM fitting result for $s_{a}^{2}=0.01$ and $s_{m}^{2}=0.99$

(a) Fitted GLM parameter $\boldsymbol{q}^{*}$

\begin{tabular}{ll}
\hline$\lambda_{11}$ & $2.22637926 \mathrm{e}-01$ \\
$\lambda_{12}$ & $2.00000000 \mathrm{e}+00$ \\
$\lambda_{22}$ & $2.87570863 \mathrm{e}-01$ \\
$\kappa$ & $5.10748491 \mathrm{e}-01$ \\
\hline$E\left(\boldsymbol{q}^{*}\right)$ & $5.88203091 \mathrm{e}-02$ \\
\hline
\end{tabular}

(b) External parameter values from $\bar{p}$

\begin{tabular}{lr}
\hline $\bar{\mu}_{1}$ & $-1.47555100 \mathrm{e}-05$ \\
$\bar{\mu}_{2}$ & $-1.56345300 \mathrm{e}-06$ \\
$\bar{\sigma}_{11}$ & $1.69044700 \mathrm{e}-02$ \\
$\bar{\sigma}_{12}$ & $2.03478000 \mathrm{e}-06$ \\
$\bar{\sigma}_{22}$ & $1.90911200 \mathrm{e}-02$ \\
\hline & "XeY" denotes $\mathrm{X} \times 10$
\end{tabular}

$$
\left(\boldsymbol{\Sigma}_{\mathcal{L}}\right)_{i j}=\frac{(\overline{\boldsymbol{\Sigma}})_{i j}}{\kappa \lambda_{i j}+(1-\kappa)} .
$$

These obviously satisfy eq. (18).

Consequently, we can parameterize our $p_{\text {mix }}$ by

$$
p_{\text {mix }}\left(x_{1}, x_{2} ; \boldsymbol{q}\right), \quad \boldsymbol{q}:=\left(\lambda_{11}, \lambda_{12}, \lambda_{22}, \kappa\right),
$$

with the measured $\overline{\boldsymbol{\mu}}$ and $\overline{\boldsymbol{\Sigma}}$ via the formula in eq. (20). We call $\boldsymbol{q}$ a GLM parameter.

\subsection{Fitting Method and Condition}

Based on the above, we fit $p_{\text {mix }}\left(x_{1}, x_{2} ; \boldsymbol{q}\right)$ to $\bar{p}\left(x_{1}, x_{2}\right)$ by solving the optimization problem:

$$
\boldsymbol{q}^{*}=\underset{\boldsymbol{q}}{\arg \min } E(\boldsymbol{q}),
$$

with the RSS-type cost function:

$$
\begin{aligned}
E(\boldsymbol{q}):=\sum_{i=1}^{N_{1}} & \sum_{j=1}^{N_{2}}\left\{p_{\text {mix }}\left(x_{1}^{i}, x_{2}^{j} ; \boldsymbol{q}\right)-\bar{p}\left(x_{1}^{i}, x_{2}^{j}\right)\right\}^{2} \\
& \times\left(\sum_{i=1}^{N_{1}} \sum_{j=1}^{N_{2}} \bar{p}\left(x_{1}^{i}, x_{2}^{j}\right)^{2}\right)^{-1}
\end{aligned}
$$

where

$$
x_{k}^{i}:=x_{k}^{a}+(i-1) \frac{x_{k}^{b}-x_{k}^{a}}{N_{k}-1}, i=1, \cdots, N_{k}, k=1,2,
$$

$N_{k}$ denotes the number of grids along $k$ th direction, and $\left[x_{1}^{a}, x_{1}^{b}\right] \times\left[x_{2}^{a}, x_{2}^{b}\right]$ is the domain of $\bar{p}\left(x_{1}, x_{2}\right)$.

Since this problem has strong nonlinearity with respect to $\boldsymbol{q}$, we solve it by means of particle swarm optimization[11,12].

\subsection{Fitting Results}

Table 1 shows the GLM fitting result of $\boldsymbol{q}^{*}$. The resulting $p_{\text {mix }}\left(x_{1}, x_{2} ; \boldsymbol{q}^{*}\right)$ is shown in Fig. 2 , plotted as its marginal PDFs. It is clear that the fitting is drastically improved as compared with those in Fig. 1 . The resulting $E\left(\boldsymbol{q}^{*}\right) \approx 0.0588$ indicates that over $94.1 \%$ of the PDF-fitness: 


- GLM fitted $\quad \circ$ PDF data
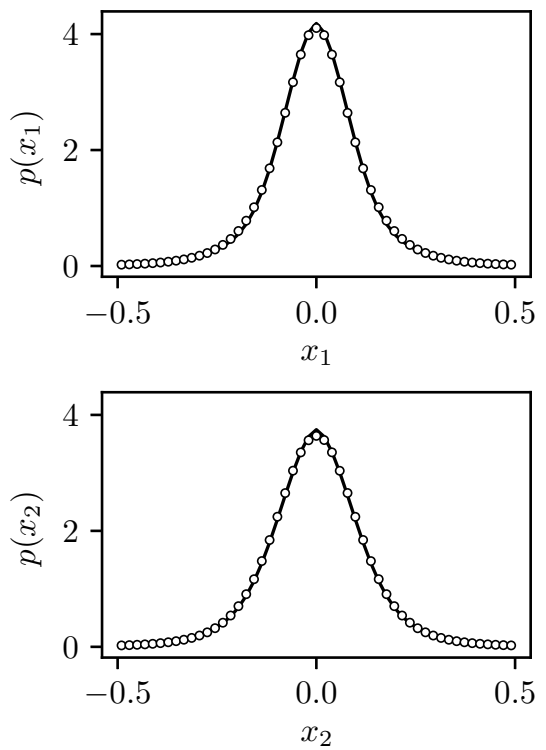

Fig. 2 The GLM model $p_{\text {mix }}\left(x_{1}, x_{2} ; \boldsymbol{q}^{*}\right)$ fitted to the PDF data of $s_{a}^{2}=0.01$ and $s_{m}^{2}=0.99$, plotted as their marginal PDFs with respect to $x_{1}$ and $x_{2}$. The PDF-fitness marks over $94.1 \%$.

$$
\left\{1-E\left(\boldsymbol{q}^{*}\right)\right\} \times 100 \%
$$

was obtained by our GLM approach.

Furthermore, the resulting mixture-ratio $\kappa \approx 0.51$ clearly explains an intermediate feature of $\bar{p}\left(x_{1}, x_{2}\right)$, indicating that probabilistic volume of $\bar{p}$ (= unity) was mostly equally divided into Gaussian and Laplacian PDFs in this case.

\section{Application to Moment-response Prediction}

In this final section, we demonstrate an application of the fitted $p_{\text {mix }}\left(x_{1}, x_{2} ; \boldsymbol{q}^{*}\right)$ to improving a stochastic equivalent linearization (SEL) technique of moment differential equations (MDEs).

\subsection{Nonlinear MDE and Equivalent Gains}

By the help of Itô calculus[13], the MDE of eq. (2) is derived in the following form:

$$
\begin{aligned}
& \frac{d \boldsymbol{m}}{d t}= \\
& {\left[\begin{array}{l}
m_{2} \\
-k m_{1}-c m_{2}-b\left\langle x_{1}^{3}\right\rangle \\
2 m_{4} \\
-k m_{3}-c m_{4}+m_{5}+b m_{1}\left\langle x_{1}^{3}\right\rangle-b\left\langle x_{1}^{4}\right\rangle \\
-2 k m_{4}-2 c m_{5}+2 b m_{2}\left\langle x_{1}^{3}\right\rangle \\
\quad-2 b\left\langle x_{1}^{3} x_{2}\right\rangle+\left(m_{1}^{2}+m_{3}\right) s_{m}^{2}+s_{a}^{2}
\end{array}\right],}
\end{aligned}
$$

where $\boldsymbol{m}:=\left(\mu_{1}, \mu_{2}, \sigma_{11}, \sigma_{12}, \sigma_{22}\right)^{T}$.

Unfortunately, this MDE cannot be solved for $\boldsymbol{m}$ as it contains nonlinear terms $\left\langle x_{1}^{3}\right\rangle,\left\langle x_{1}^{4}\right\rangle$, and $\left\langle x_{1}^{3} x_{2}\right\rangle$. Extending $\boldsymbol{m}$ to contain such higher moments does
Table 2 SEL gains based on $\mathcal{N}$ and $\mathcal{L}$

\begin{tabular}{c|cc}
$h\left(x_{1}, x_{2}\right)$ & $\varphi[\mathcal{N}, h]$ & $\varphi[\mathcal{L}, h]$ \\
\hline$x_{1}^{3}$ & $3 \mu_{1} \sigma_{11}$ & $3 \mu_{1} \sigma_{11}$ \\
\hline$x_{1}^{3} x_{2}$ & $\sigma_{11}\left(6 \mu_{1}^{2}+3 \sigma_{11}\right)$ & $\sigma_{11}\left(6 \mu_{1}^{2}+5 \sigma_{11}\right)$ \\
\hline$x_{1}^{4}$ & $3 \mu_{1} \mu_{2} \sigma_{11}+3 \mu_{1}^{2} \sigma_{12}$ & $3 \mu_{1} \mu_{2} \sigma_{11}+3 \mu_{1}^{2} \sigma_{12}$ \\
& $+3 \sigma_{11} \sigma_{12}$ & $+5 \sigma_{11} \sigma_{12}$
\end{tabular}

not work because this causes more higher moments. Therefore, there is no choice but to solve the MDE in eq. (26) approximately. SEL techniques[14-17] provide one of the fundamental bases of such approximations[13,18-20].

In a standard SEL approach, a Gaussian distribution is assumed to approximate nonlinear terms by

$$
\begin{aligned}
& \left\langle h\left(x_{1}, x_{2}\right)\right\rangle \approx \varphi[\mathcal{N}, h](\boldsymbol{m}) \\
& \quad:=\iint_{-\infty}^{\infty} h\left(x_{1}, x_{2}\right) \mathcal{N}\left(x_{1}, x_{2} ; \boldsymbol{\mu}, \boldsymbol{\Sigma}_{\mathcal{N}}\right) d x_{1} d x_{2}
\end{aligned}
$$

where $h\left(x_{1}, x_{2}\right)$ is a scalar-valued function of random variables. As $\mathcal{N}$ is a function of $\boldsymbol{m}$, the result becomes a scalar-valued function $\varphi[\mathcal{N}, h](\boldsymbol{m})$ of $\boldsymbol{m}$, which is called a SEL gain. This approximation works well only if $\boldsymbol{x}$ is almost Gaussian. One can replace $\mathcal{N}$ with $\mathcal{L}$ as the Laplacian distribution $\mathcal{L}$ is also parameterized by $\boldsymbol{m}$. This also results in a scalar-valued function of $\boldsymbol{m}$ and works well only if $\boldsymbol{x}$ is almost Laplacian.

Table 2 lists the resulting SEL gains derived based on the Gaussian and Laplacian distributions via the moment-generating functions in eqs. (13), (14), i.e.,

$$
\varphi\left[\mathcal{P}, x_{1}^{k} x_{2}^{l}\right](\boldsymbol{m})=\left.\frac{\partial^{k+l} M_{\mathcal{P}}\left(t_{1}, t_{2}\right)}{\partial t_{1}^{k} \partial t_{2}^{l}}\right|_{\substack{t_{1}=0 \\ t_{2}=0}}
$$

for $\mathcal{P}=\mathcal{N}, \mathcal{L}$.

\subsection{GLM Equivalent Gains}

The SEL gains in Table 2 are not expected to provide good approximation of the PDF data as its distribution resembles neither the Gaussian or Laplacian distributions. Instead, we use the well-fitted $p_{\text {mix }}\left(x_{1}, x_{2} ; \boldsymbol{q}^{*}\right)$ in Fig. 2 to propose a GLM equivalent gain in the following form:

$$
\begin{aligned}
& \varphi\left[p_{\text {mix }}, h\right](\boldsymbol{m}) \\
& :=\iint_{-\infty}^{\infty} h\left(x_{1}, x_{2}\right) p_{\text {mix }}\left(x_{1}, x_{2} ; \boldsymbol{q}^{*}\right) d x_{1} d x_{2} \\
& =\kappa \varphi[\mathcal{N}, h]\left(\boldsymbol{m}_{\mathcal{N}}\right)+(1-\kappa) \varphi[\mathcal{L}, h]\left(\boldsymbol{m}_{\mathcal{L}}\right) .
\end{aligned}
$$

Here, different moment vectors $\boldsymbol{m}_{\mathcal{N}}$ and $\boldsymbol{m}_{\mathcal{L}}$ are introduced. These are to maintain our distributive formulation using covariance-ratio $\lambda_{i j}$ in eq. (20). Specifically, the solution $\boldsymbol{m}$ of eq. (26) are distributed to $\boldsymbol{m}_{\mathcal{N}}=\left(\mu_{1}^{\mathcal{N}}, \mu_{2}^{\mathcal{N}}, \sigma_{11}^{\mathcal{N}}, \sigma_{12}^{\mathcal{N}}, \sigma_{22}^{\mathcal{N}}\right)^{T}$ and $\boldsymbol{m}_{\mathcal{L}}=$ $\left(\mu_{1}^{\mathcal{L}}, \mu_{2}^{\mathcal{L}}, \sigma_{11}^{\mathcal{L}}, \sigma_{12}^{\mathcal{L}}, \sigma_{22}^{\mathcal{L}}\right)^{T}$ in the following manner:

$$
\begin{aligned}
& \mu_{i}^{\mathcal{N}}=\mu_{i}^{\mathcal{L}}=\mu_{i}, \\
& \sigma_{i j}^{\mathcal{N}}=\frac{\lambda_{i j} \sigma_{i j}}{\kappa \lambda_{i j}+(1-\kappa)},
\end{aligned}
$$




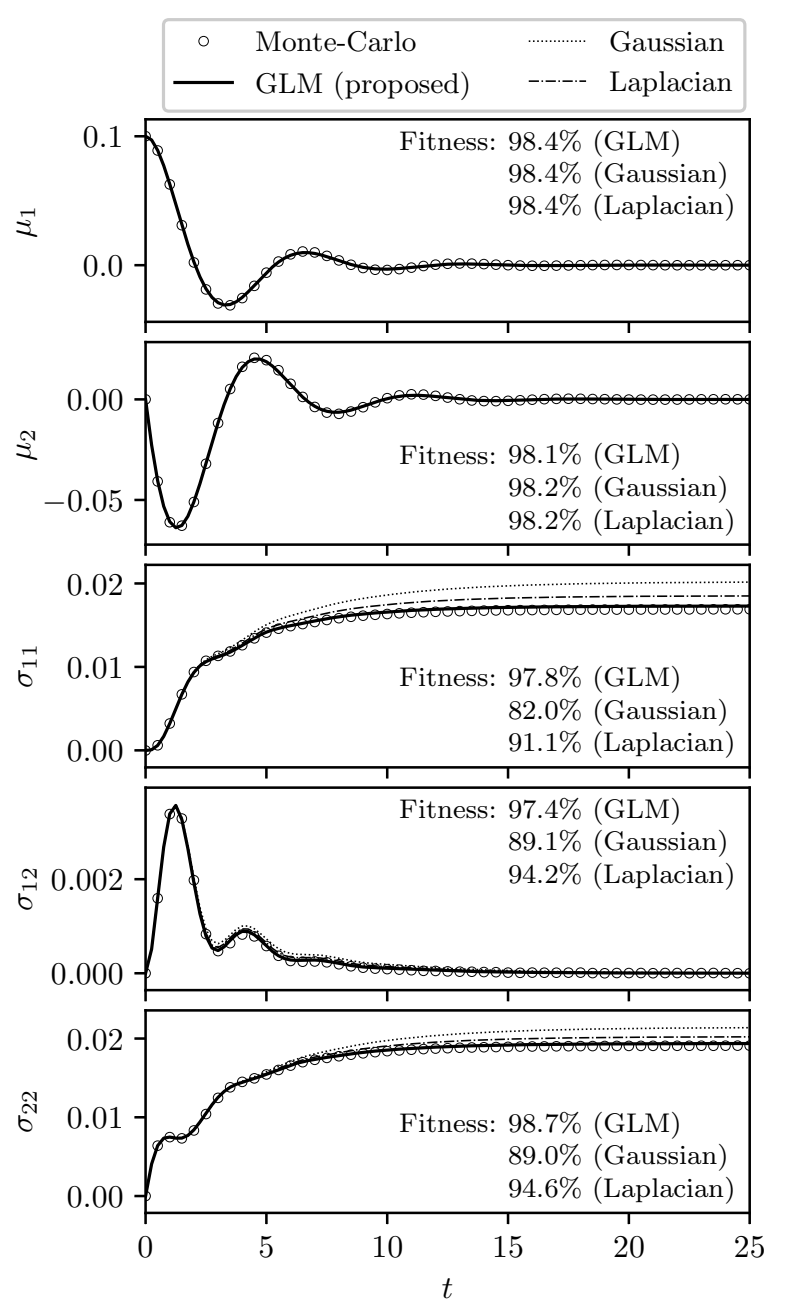

Fig. 3 Transient moment responses predicted by the GLM equivalent gains fitted to the PDF data of $s_{a}^{2}=0.01$ and $s_{m}^{2}=0.99$.

$$
\sigma_{i j}^{\mathcal{L}}=\frac{\sigma_{i j}}{\kappa \lambda_{i j}+(1-\kappa)},
$$

for $1 \leq i \leq j \leq 2$.

Therefore, we have the GLM equivalent gains that approximate the nonlinear terms as follows.

$$
\begin{aligned}
& \left\langle x_{1}^{3}\right\rangle \approx \varphi\left[p_{\text {mix }}, x_{1}^{3}\right](\boldsymbol{m}), \\
& \left\langle x_{1}^{4}\right\rangle \approx \varphi\left[p_{\text {mix }}, x_{1}^{4}\right](\boldsymbol{m}), \\
& \left\langle x_{1}^{3} x_{2}\right\rangle \approx \varphi\left[p_{\text {mix }}, x_{1}^{3} x_{2}\right](\boldsymbol{m}) .
\end{aligned}
$$

\subsection{Moment-response Prediction}

In Fig. 3, the solid lines indicate the numerical solutions of MDE in eq. (26) using the GLM equivalent gains in eq. (31) and the small circles indicate the Monte-Carlo simulation results obtained from the original Duffing oscillator in eq. (2). The dotted and broken lines indicate the MDE solutions using the conventional Gaussian SEL[16,21] and Laplacian SEL gains listed in Table 2, respectively. Furthermore, the values shown in each graph indicate the RSS fitness values of these MDE solutions to the corresponding Monte-Carlo simulation results. We call them $M D E$ -
Table 3 GLM fitting result for $s_{a}^{2}=0.5$ and $s_{m}^{2}=0.5$

(a) Fitted GLM parameter $\boldsymbol{q}^{*}$

\begin{tabular}{ll}
\hline$\lambda_{11}$ & $1.59720389 \mathrm{e}+00$ \\
$\lambda_{12}$ & $4.59757281 \mathrm{e}-06$ \\
$\lambda_{22}$ & $4.22487100 \mathrm{e}-02$ \\
$\kappa$ & $9.96142446 \mathrm{e}-01$ \\
\hline$E\left(\boldsymbol{q}^{*}\right)$ & $4.37725730 \mathrm{e}-02$ \\
\hline
\end{tabular}

(b) External parameter values from $\bar{p}$

\begin{tabular}{lr}
\hline $\bar{\mu}_{1}$ & $-3.07931000 \mathrm{e}-05$ \\
$\bar{\mu}_{2}$ & $-1.33121000 \mathrm{e}-05$ \\
$\bar{\sigma}_{11}$ & $2.62309800 \mathrm{e}-01$ \\
$\bar{\sigma}_{12}$ & $-1.26887800 \mathrm{e}-05$ \\
$\bar{\sigma}_{22}$ & $4.50878600 \mathrm{e}-01$ \\
\hline & "XeY" denotes $\mathrm{X} \times 10^{\mathrm{Y}}$
\end{tabular}

fitness.

These results clearly show that the best-fitting moment responses were obtained by our GLM approach that marks over $97.3 \%$ MDE-fitness over all components of $\boldsymbol{m}(t)$. The superiority of GLM was conspicuous especially for covariances $\sigma_{11}, \sigma_{12}$, and $\sigma_{22}$. The second performance is obtained by Laplacian SEL and the worst by conventional Gaussian SEL.

Next, we demonstrate the applicability of our method to another PDF data having different characteristics. Table 3 shows the GLM fitting result to the PDF data for $s_{a}^{2}=0.5$ and $s_{m}^{2}=0.5$, i.e., using stronger-additive and weaker-multiplicative noise intensities. The resulting $E\left(\boldsymbol{q}^{*}\right) \approx 0.0438$ indicates over 95.6\% PDF-fitness, which is slightly better than the preceding case in Table 1.

The resulting mixture-ratio $\kappa>0.96$ explains that this PDF data is almost Gaussian. The corresponding moment-response prediction results are shown in Fig. 4. As indicated by the $\kappa$-value nearly unity, our GLM prediction (solid lines) and the Gaussian prediction (dotted lines) fitted the Monte-Carlo results (small circles), at the same level of accuracy (although tiny discrepancy appeared in $\sigma_{12}$ ). In contrast, the Laplacian prediction resulted in the worst fitness in each case.

Furthermore, Table 4 summarizes the fitting results for other $\left(s_{a}^{2}, s_{m}^{2}\right)$ conditions, including those already obtained in Tables 1, 3 and Figs. 3, 4 (labeled by (a) and (c)). The Frobenius norm of the covariance matrix $|\overline{\boldsymbol{\Sigma}}|$ of each PDF data is also listed.

First, as the relative additive-strength: $s_{a}^{2} / s_{m}^{2}$ increased, the mixture-ratio $\kappa$ monotonically increased toward unity (maximum), indicating that our GLM tended to be almost Gaussian or less heavy-tailed. In this process, the PDF-fitness was rapidly getting worse near $\kappa=1$ (from Table 4 (c) to (d) and (e)); at the same time, $|\bar{\Sigma}|$ increased, indicating that the distribution of $x_{1}$ and $x_{2}$ was expanded. This implies that, as $s_{a}^{2} / s_{m}^{2}$ increased, our GLM tended to be al- 


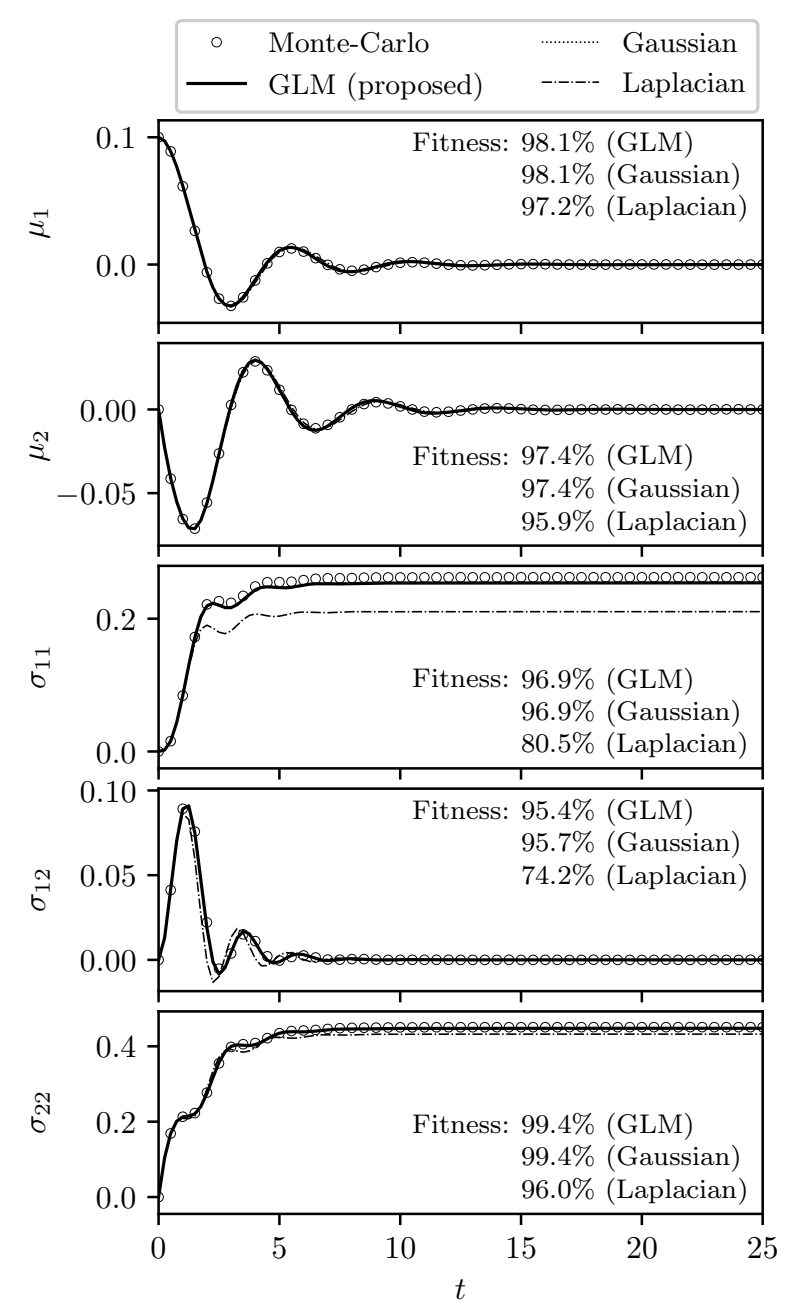

Fig. 4 Transient moment responses predicted by the GLM equivalent gains fitted to the PDF data of $s_{a}^{2}=0.5$ and $s_{m}^{2}=0.5$.

most Gaussian and the Gaussian SEL approximation tended to be suffered from the system's nonlinearity emphasized by the largely distributed states. Unfortunately, such nonlinear effects are hardly avoidable as long as using SEL techniques.

Then, we focus on the well PDF-fitted cases (a), (b), and (c); they resulted in over 94\% PDF-fitness. In these cases, our GLM and SEL-gains yielded over 90\% MDE-fitness and they did not induce big failure, unlike the Gaussian $\sigma_{11}, \sigma_{12}$, and $\sigma_{22}$ in Table 4 (a) and the Laplacian $\sigma_{11}$ and $\sigma_{12}$ in Table 4 (c).

The above results suggest that our GLM-based PDF model and SEL gains can provide a robust approximation of heavy-tailed PDF data when they have weak nonlinearity, with the GLM parameters properly adjusted.

\section{Conclusion}

In this study, we have proposed a GLM-type PDF model to describe heavy-tailed PDF data generated by a nonlinear vibration system subjected to both additive and multiplicative random excitations.
Table 4 Fitting results for other $\left(s_{a}^{2}, s_{m}^{2}\right)$ conditions. Percentage values denote the MDE-fitness values via different SELs. $|\overline{\boldsymbol{\Sigma}}|$ denotes Frobenius norm of the covariance matrix of the PDF data

(a) $\left(s_{a}^{2}, s_{m}^{2}\right)=(0.01,0.99): \quad$ PDF-fitness $=94.1 \%$, \begin{tabular}{ccccc}
$\kappa=5.10748491 \mathrm{e}-01$, & $|\bar{\Sigma}|=2.54996466 \mathrm{e}-02$ \\
\hline
\end{tabular}

\begin{tabular}{llllll}
\hline (MDE-fitness) & $\mu_{1}$ & $\mu_{2}$ & $\sigma_{11}$ & $\sigma_{12}$ & $\sigma_{22}$ \\
GLM & $98.4 \%$ & $98.1 \%$ & $97.8 \%$ & $97.4 \%$ & $98.7 \%$
\end{tabular}

$\begin{array}{lllllll}\text { Gaussian } & 98.4 \% & 98.2 \% & 82.0 \% & 89.1 \% & 89.0 \%\end{array}$ \begin{tabular}{llllll} 
Laplacian & $98.4 \%$ & $98.2 \%$ & $91.1 \%$ & $94.2 \%$ & $94.6 \%$ \\
\hline
\end{tabular} (b) $\left(s_{a}^{2}, s_{m}^{2}\right)=(0.1,0.9): \quad$ PDF-fitness $=94.4 \%$, $\kappa=6.66800063 \mathrm{e}-01, \quad|\bar{\Sigma}|=1.66393224 \mathrm{e}-01$ \begin{tabular}{llllll}
\hline (MDE-fitness) & $\mu_{1}$ & $\mu_{2}$ & $\sigma_{11}$ & $\sigma_{12}$ & $\sigma_{22}$
\end{tabular}

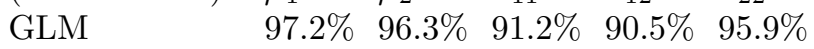
$\begin{array}{llllll}\text { Gaussian } & 97.4 \% & 96.8 \% & 92.3 \% & 92.8 \% & 96.5 \%\end{array}$ \begin{tabular}{llllll} 
Laplacian & $97.2 \%$ & $96.3 \%$ & $91.0 \%$ & $92.1 \%$ & $95.8 \%$ \\
\hline
\end{tabular} (c) $\left(s_{a}^{2}, s_{m}^{2}\right)=(0.5,0.5): \quad$ PDF-fitness $=95.6 \%$, $\kappa=9.96142446 \mathrm{e}-01, \quad|\bar{\Sigma}|=5.21630083 \mathrm{e}-01$ \begin{tabular}{llllll}
\hline (MDE-fitness) & $\mu_{1}$ & $\mu_{2}$ & $\sigma_{11}$ & $\sigma_{12}$ & $\sigma_{22}$
\end{tabular} $\begin{array}{llllll}\text { GLM } & 98.1 \% & 97.4 \% & 96.9 \% & 95.4 \% & 99.4 \%\end{array}$ $\begin{array}{llllll}\text { Gaussian } & 98.1 \% & 97.4 \% & 96.9 \% & 95.7 \% & 99.4 \%\end{array}$ \begin{tabular}{llllll} 
Laplacian & $97.2 \%$ & $95.9 \%$ & $80.5 \%$ & $74.2 \%$ & $96.0 \%$ \\
\hline
\end{tabular} (d) $\left(s_{a}^{2}, s_{m}^{2}\right)=(0.9,0.1): \quad$ PDF-fitness $=91.7 \%$, $\kappa=9.99954602 \mathrm{e}-01, \quad|\bar{\Sigma}|=7.55872456 \mathrm{e}-01$ \begin{tabular}{llllll}
\hline (MDE-fitness) & $\mu_{1}$ & $\mu_{2}$ & $\sigma_{11}$ & $\sigma_{12}$ & $\sigma_{22}$
\end{tabular} $\begin{array}{llllll}\text { GLM } & 94.7 \% & 91.8 \% & 94.1 \% & 86.9 \% & 99.5 \%\end{array}$ $\begin{array}{lllllll}\text { Gaussian } & 94.7 \% & 91.8 \% & 94.1 \% & 86.9 \% & 99.5 \%\end{array}$ \begin{tabular}{llllll} 
Laplacian & $94.0 \%$ & $91.6 \%$ & $78.4 \%$ & $62.6 \%$ & $98.6 \%$ \\
\hline
\end{tabular} (e) $\left(s_{a}^{2}, s_{m}^{2}\right)=(0.99,0.01): \quad$ PDF-fitness $=90.7 \%$, $\kappa=9.99999998 \mathrm{e}-01, \quad|\bar{\Sigma}|=8.00366724 \mathrm{e}-01$ \begin{tabular}{llllll}
\hline (MDE-fitness) & $\mu_{1}$ & $\mu_{2}$ & $\sigma_{11}$ & $\sigma_{12}$ & $\sigma_{22}$
\end{tabular}

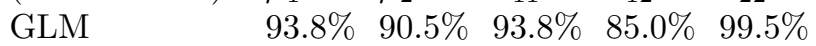
$\begin{array}{lllllll}\text { Gaussian } & 93.8 \% & 90.5 \% & 93.8 \% & 85.0 \% & 99.5 \%\end{array}$ $\begin{array}{llllll}\text { Laplacian } & 93.2 \% & 90.5 \% & 78.2 \% & 60.3 \% & 98.7 \%\end{array}$ "XeY" denotes $\mathrm{X} \times 10^{\mathrm{Y}}$

First, we proposed a GLM-type PDF model $p_{\text {mix }}$ as a convex combination of the Gaussian and Laplacian PDFs with mixture-ratio $\kappa$. Then, we developed a method to fit the proposed $p_{\text {mix }}$ to the PDF data using the four-dimensional GLM parameter $\boldsymbol{q}$, formulating the optimization problem to minimize the RSS between our $p_{\text {mix }}$ and the PDF data. The results showed the following.

- Our proposed $p_{\text {mix }}$ drastically improved the PDF-fitness to the heavy-tailed PDF data as compared with separate Gaussian and Laplacian PDFs.

- In such cases, the resulting PDF-fitness of our proposed $p_{\text {mix }}$ was over $94 \%$.

Next, we applied the resulting $p_{\text {mix }}$ to improve accuracy of SEL and obtained the following results.

- Our proposed GLM equivalent gains yielded robust fittings of moment responses $\boldsymbol{m}(t)$ as compared with those by conventional SEL methods.

- Especially for the heavy-tailed PDF data of 
week nonlinearity, the resulting MDE-fitness of moment responses was over $90 \%$ for all components of $\boldsymbol{m}(t)$.

Given the above, we believe that our GLM approach provides a simple but effective way to approximate heavy-tailed PDF data.

In future, we plan to investigate the applicability of our GLM to real-world heavy-tailed fluctuations.

\section{Acknowledgements}

This work was funded by JSPS KAKENHI Grant Numbers JP18H01391 and JP17H06552.

\section{References}

[1] V. Pisarenko and M. Rodkin: Heavy-Tailed Distributions in Disaster Analysis, Advances in Natural and Technological Hazards Research, Springer Netherlands (2010)

[2] S. Rachev: Handbook of Heavy Tailed Distributions in Finance: Handbooks in Finance, Elsevier Science (2003)

[3] J. L. Cabrera and J. G. Milton: Human stick balancing: tuning Lèvy flights to improve balance control; Chaos, Vol. 14, No. 3, pp. 691-698 (2004)

[4] J. Cabrera and J. Milton: Stick balancing: On-off intermittency and survival times; Nonlinear Studies, Vol. 11, No. 3, pp. 305-317 (2004).

[5] S. Matsumoto, K. Yoshida and M. Sekikawa: Stochastic dynamic modeling of human visuomotor tracking task of an unstable virtual object; Transactions of the Institute of Systems, Control and Information Engineers, Vol. 31, No. 6, pp. 209-219 (2018)

[6] K. Sigman: Appendix: A primer on heavy-tailed distributions; Queueing Syst. Theory Appl., Vol. 33, No. 1-3, pp. 261-275 (1999)

[7] S. Asmussen, K. Binswanger and B. Hjgaard: Rare events simulation for heavy-tailed distributions; Bernoulli, Vol. 6, No. 2, pp. 303-322 (2000)

[8] P. Z. Peebles: Probability, Random Variables, and Random Signal Principles, 2nd ed, McGraw-Hill (1987)

[9] D. N. Naik and K. Plungpongpun: A Kotz-type distribution for multivariate statistical inference BT; Advances in Distribution Theory, Order Statistics, and Inference, ed. N. Balakrishnan, J.M. Sarabia, and E. Castillo, pp. 111-124, Birkhäuser Boston (2006)

[10] S. Kotz, T. Kozubowski and K. Podgorski: The Laplace Distribution and Generalizations: A Revisit with Applications to Communications, Economics, Engineering, and Finance, Progress in Mathematics Series, Birkhäuser Boston (2001)

[11] J. Kennedy and R. Eberhart: Particle swarm optimization; Proceedings of IEEE International Conference on Neural Networks, pp. 1942-1948 (1995)

[12] A. P. Engelbrecht: Fundamentals of Computational Swarm Intelligence, John Wiley \& Sons (2005)

[13] C. W. Gardiner: Handbook of Stochastic Methods for Physics, Chemistry and the Natural Sciences, third ed., Springer Series in Synergetics, Vol. 13, Springer-Verlag (2004)

[14] Y. Sawaragi, N. Sugai and Y. Sunahara: Statistical Studies of Non-linear Control Systems, Nippon Printing (1962)

[15] T. S. Atalik and S. Utku: Stochastic linearization of multi-degree-of-freedom non-linear systems; Earthquake Engineering \& Structural Dynamics, Vol. 4, No. 4, pp. 411-420 (1976)

[16] L. Socha: Statistical and equivalent linearization techniques with probability density criteria; Journal of Theoretical and Applied Mechanics, Vol. 37, No. 2, pp. 369-382 (1999)

[17] J. Roberts and P. Spanos: Random Vibration and Statistical Linearization; Dover Civil and Mechanical Engineering Series, Dover Publications (2003)

[18] D. Bover: Moment equation methods for nonlinear stochastic systems; Journal of Mathematical Analysis and Applications, Vol. 65, No. 2, pp. 306-320 (1978)

[19] Y. Lin and G. Cai: Probabilistic Structural Dynamics, Mcgraw Hill Book Company (2004)

[20] H. Singer: Moment equations and Hermite expansion for nonlinear stochastic differential equations with application to stock price models; Computational Statistics, Vol. 21, pp. 385-397 (2006)

[21] K. Yoshida and A. Higeta: Toward stochastic explanation of a neutrally stable delayed feedback model of human balance control; Int. J Innovative Computing, Information and Control, Vol. 8, No. 3, pp. 2249-2259(2012)

\section{Authors}

Katsutoshi Yoshida (Member)

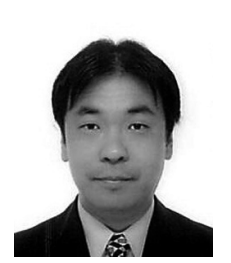

Katsutoshi Yoshida received the Ph.D. degree in Engineering from Utsunomiya University, Utsunomiya, Japan, in 1996. Since 2012, he is Professor at the Graduate School of Engineering, Utsunomiya University. His research interests are in nonlinear dynamics, stochastic mechanics, complex systems, and multi-human dynamics. He is a member of JSME and ISCIE.

\section{Yoshikazu YamanakA}

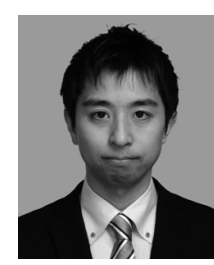

Yoshikazu Yamanaka received the Ph.D. degree in Engineering from Nagaoka University of Technology, Niigata, Japan, in 2017. Since 2017, he is Assistant Professor at the Graduate School of Engineering, Utsunomiya University. His research interests are in evolutionary computation, swarm intelligence, and nonlinear dynamics. He is a member of IEICE and IEEE. 